\title{
Oscillatory combustion waves in a chain branching model
}
H. S. Sidhu ${ }^{1}$
V. V. Gubernov ${ }^{2}$
G. N. Mercer $^{5}$
A. V. Kolobov ${ }^{3}$
A. A. Polezhaev ${ }^{4}$
J. J. Sharples ${ }^{6}$

(Received 15 August 2008; revised 1 July 2009)

\begin{abstract}
We consider an adiabatic model with a two step chain branching reaction mechanism, and use a numerical scheme to show that as the parameter characterizing the fuel properties is increased, the combustion waves begin to exhibit oscillatory behaviour. We show that this behaviour becomes more complex as the fuel parameter is further increased until extinction of the combustion reaction occurs.
\end{abstract}

\section{Contents}

1 Introduction

C1018

2 Mathematical formulation

C1020

3 Travelling wave solution

C1021

http://anziamj.austms.org.au/ojs/index.php/ANZIAMJ/article/view/1467 gives this article, (c) Austral. Mathematical Soc. 2009. Published July 30, 2009. ISSN 1446-8735. (Print two pages per sheet of paper.) 


\section{Introduction}

Reaction-diffusion models designed to simulate combustion processes have been studied by researchers over a long period of time [8]. Travelling wave solutions of these models (known as combustion waves) describe a propagating flame front, which marks the transition from the initial mixture to the reactant product equilibrium phase. Combustion waves have been observed in a number of experiments [8] and are of considerable importance in industrial processes, such as those used to synthesise advanced materials. While combustion models incorporating only basic one step kinetic schemes have been extremely useful in predicting behaviour that is qualitatively correct, more accurate descriptions require the inclusion of more complex chemical kinetics. Consequently, a number of reaction schemes involving multi-step kinetics appear in the literature. One such example is the two step, chain branching model introduced by Dold and Weber [2].

Gubernov et al. [6] and Sidhu et al. [9] investigated properties of the model introduced by Dold and Weber [2] in the adiabatic case and in the limit of equal diffusivity of the reactant, the radical and heat. In contrast to Dold and Weber [2], Gubernov et al. [6] and Sidhu et al. [9] assume that the activation energy is $\mathrm{O}(1)$ (not an infinite number). As noted by Mikolaitis [7], this is a reasonable assumption for real flames like the hydrogen oxidation flame. We also used a different nondimensionalization, which enabled us to make more convenient comparisons between the two and one step models. Gubernov et al. [6] and Sidhu et al. [9] investigated in detail the properties of travelling wave solutions using numerical simulation. The numerical sim- 
ulations demonstrated that the speed of a combustion wave as a function of parameters is single valued. We found that for finite activation energy there is a residual amount of reactant left behind the travelling combustion wave which is not used in the reaction [4]. This makes the problem similar to the nonadiabatic one step premixed flames. The other characteristic of the model considered by Gubernov, Sidhu et al. [6, 9], which makes the similarity between the adiabatic two step reaction and the nonadiabatic one step system even stronger, is that for certain parameter values the combustion wave exhibits extinction. However, for the former case the extinction occurs at zero flame speed. This mainly distinguishes the one and two step adiabatic models. The route to extinction in this model was investigated in detail by Gubernov et al. [5]. They showed that the flame speed as a function of activation energy approaches zero in a linear fashion. The stability of the travelling combustion waves was also investigated: for the equidiffusional case the flame is stable over the wide range of parameter values considered in the article; a result that correlates with the results of Dold and Weber [2].

The equidiffusional approximation used in our earlier articles [5, 6, 9] makes the analysis of the problem more convenient. However, this distinguished limit reduces the applicability of the results to real flames with chain branching reaction mechanism, which are characterized by various values of Lewis numbers for both radicals, $\mathrm{L}_{B}$, and fuel, $\mathrm{L}_{\mathrm{A}}$. This is especially true for the stability analysis, since flame stability is expected to depend substantially on these parameters [2].

Recently, Gubernov et al. [5] investigated the effect of Lewis number variation on both the properties and stability of combustion waves in this model. They showed that the Lewis number for fuel has a significant effect on the properties and stability of premixed flames, whereas variation of the Lewis number for the radicals has only quantitative (but not qualitative) effects on the combustion waves. We demonstrate that, when the Lewis number for fuel is less than unity, the flame speed is a unique, monotonically decreasing function of the dimensionless activation energy. The combustion wave is stable and exhibits extinction for finite values of activation energy as the flame 
speed decreases to zero. For fuel Lewis number greater than unity, the flame speed is a C-shaped and double valued function. The slow solution branch is shown to be unstable whereas the fast solution branch is stable or exhibits the onset of pulsating instabilities via the Hopf bifurcation. However, for the parameter values used in our study, no oscillatory combustion waves were detected.

The aim in this study is to obtain a detailed map of the onset of pulsating instabilities in this reaction scheme. This includes the analysis of the various bifurcations and scenarios leading to instabilities. In particular, we study the properties of the Hopf bifurcation leading to the onset of pulsations in detail and investigate the pulsating solutions emerging as a result of this bifurcation. In the past, both theoretical work, such as that of Bayliss and Matkowsky [1] and Weber et al. [10], and experimental investigations, such as that of Feng et al. [3], confirmed the existence of such pulsating combustion phenomena.

\section{Mathematical formulation}

We consider an adiabatic model in one spatial dimension that includes two steps:

$$
\begin{aligned}
\text { autocatalytic chain branching, } \quad \mathrm{A}+\mathrm{B} & \rightarrow 2 \mathrm{~B} ; \\
\text { recombination, } \mathrm{B}+\mathrm{M} & \rightarrow \mathrm{C}+\mathrm{M} .
\end{aligned}
$$

Here $A$ is the fuel, $B$ is the radical, $C$ is the product, and $M$ represents any molecule that is required to start the reaction but remains unchanged by the reaction. As in previous work [5, 9], the governing equations for the nondimensional temperature, $u$, concentration of fuel, $v$, and radicals, $w$, are written in nondimensional form as

$$
u_{t}=u_{x x}+r w,
$$




$$
\begin{aligned}
v_{\mathrm{t}} & =\mathrm{L}_{\mathrm{A}}^{-1} v_{x x}-\beta v w e^{-1 / u}, \\
w_{\mathrm{t}} & =\mathrm{L}_{\mathrm{B}}^{-1} w_{x x}+\beta v w e^{-1 / u}-\mathrm{r} \beta w
\end{aligned}
$$

where $x$ and $t$ are the dimensionless spatial coordinate and time respectively, $L_{A}$ and $L_{B}$ are the Lewis numbers for fuel and radicals respectively, $\beta$ is the dimensionless activation energy of the chain branching step (which corresponds to the definition for the one step model [4]), $r$ is the ratio of the characteristic time of the recombination and branching steps (which cannot be reproduced in one step approximations of the flame kinetics).

Equations (2)-(4) are considered subject to the boundary conditions

$$
\begin{aligned}
& u=0, \quad v=1, \quad w=0 \quad \text { for } x \rightarrow+\infty \\
& u_{x}=0, \quad v_{x}=0, \quad w=0 \text { for } x \rightarrow-\infty
\end{aligned}
$$

On the right boundary $(x \rightarrow+\infty)$ we have cold $(u=0)$ and unburned state $(v=1)$, where the fuel has not been consumed yet and no radicals have been produced $(w=0)$. On the left boundary $(x \rightarrow-\infty)$ neither the temperature of the mixture nor the concentration of fuel can be specified. We only require that there is no reaction occurring so the solution reaches a steady state of (2)-(4). Therefore the derivatives of $\boldsymbol{u}$ and $\boldsymbol{v}$ are zero and $w=0$ for $x \rightarrow-\infty$.

\section{Travelling wave solution}

We seek a solution to the problem (2)-(6) in the form of a travelling wave $u(x, t)=u(\xi), v(x, t)=v(\xi)$, and $w(x, t)=w(\xi)$, where the coordinate in the moving frame $\xi=x-c t$, and $c$ is the speed of the travelling wave. Substituting this travelling wave solution into the governing equations we obtain

$$
u_{\xi \xi}+c u_{\xi}+r w=0
$$




$$
\begin{aligned}
& \mathrm{L}_{A}^{-1} v_{\xi \xi}+c v_{\xi}-\beta v w e^{-1 / u}=0, \\
& \mathrm{~L}_{\mathrm{B}}^{-1} w_{\xi \xi}+c w_{\xi}+\beta v w e^{-1 / u}-r \beta w=0,
\end{aligned}
$$

and boundary conditions

$$
\begin{aligned}
u=0, & v=1, \quad w=0 \quad \text { for } \xi \rightarrow+\infty \\
u_{\xi}=0, & v_{\xi}=0, \quad w=0 \quad \text { for } \xi \rightarrow-\infty .
\end{aligned}
$$

The properties of the travelling combustion waves are investigated numerically by solving the system of ordinary differential equations (7)-(9) subject to boundary conditions (11). We use a standard shooting algorithm with a fourth order Runge-Kutta integration scheme in the first instance and then correct the results by employing the relaxation algorithm. The investigation of the travelling combustion waves described by Gubernov et al. [5], using the methods described above, revealed that the Lewis number for fuel $L_{A}$ has a substantial effect on the properties of the premixed flames, whereas the variation of $L_{B}$ and $r$ affects only quantitative behavior of the combustion waves. The results obtained by Gubernov et al. [5] and in the course of our current work are summarized in Figure 1 where the flame speed is plotted as a function of $\beta$ for various values of $L_{A}$ and for $L_{B}=1$ and $r=0.001$. This figure captures all the generic properties of the travelling wave solution that is possible for this model. In physical terms, increasing $\beta$ implies that the fuel is less exothermic, and hence extinction of the combustion process occurs.

From Figure 1, for the case of Lewis number for fuel less than unity $\left(L_{A}<1\right)$, the dependence $c(\beta)$ is a monotonically decaying function exhibiting extinction as the flame speed reaches zero at a certain value of the activation energy, $\beta_{e}$, corresponding to extinction. The flame speed decreases to zero according to a quadratic law. However, when $L_{A}=1$ the structure of the travelling solution branch in the parameter space changes: although the dependence of the flame speed, $\mathrm{c}$, on $\beta$ is still a monotonic function approaching zero as $\beta$ reaches some critical value $\beta_{e} \approx 4.2$ corresponding to extinction, the flame speed now decreases to zero according to a linear law. 


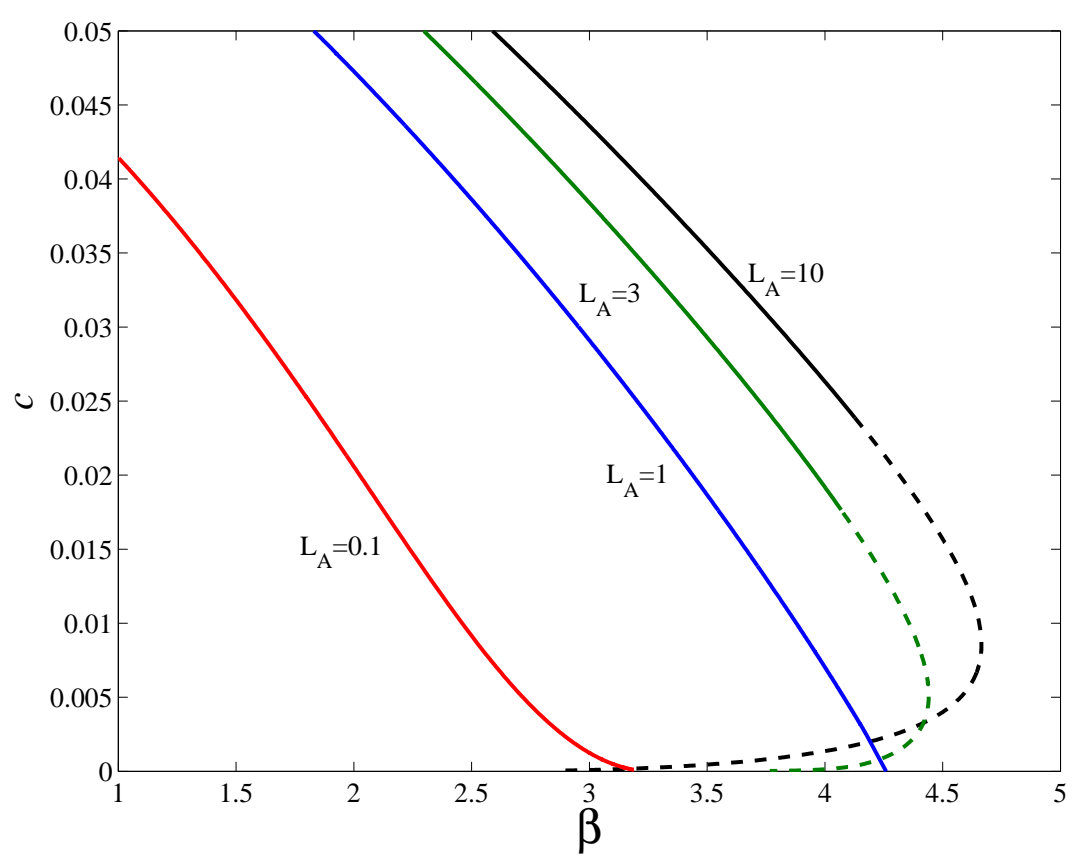

Figure 1: The dependence of the flame speed, $\mathbf{c}$, on the dimensionless activation energy, $\beta$, for four values of Lewis number $L_{A}$. Solid lines represent stable solutions, whereas unstable solutions are plotted with dashed lines. 
The dependence of the flame speed, $c$, on $\beta$ becomes C-shaped for $L_{A}>1$; that is, $c(\beta)$ is a double valued function. There are either two solutions travelling with different flame speed or the solutions cease to exist due to the extinction when the fast solution branch meets the slow solution branch at the turning point of the $c(\beta)$ curve. The solution branch also loses its stability at a Hopf bifurcation point. For the case $\mathrm{L}_{\mathrm{A}}=10$ the Hopf bifurcation occurs at $\beta \approx 4.1225$. Despite numerous attempts, we were unable to detect any pulsating solutions for $\beta>4.1225$ when $L_{A}=10$. For our present analysis, we concentrate on the case $\mathrm{L}_{A}=3$ (Figure 1). The Hopf bifurcation point for $L_{A}=3$ is located at $\beta \approx 4.0703$. The next section examines pulsating solutions for the case $L_{A}=3$.

\section{Oscillatory combustion solutions}

We investigate the properties of pulsating combustion wave solutions emerging as a result of the Hopf bifurcation when the parameters reach critical values. The governing partial differential equations (2)-(4) are solved in a sufficiently large coordinate domain with the boundary conditions (5)-(6) imposed at the edge points of the space grid. For our numerical algorithm we use the method of splitting with respect to physical processes. Initially we solve the set of ordinary differential equations which describe the temperature and the species concentration variations due to the branching and recombination reactions by using the fourth order Runge-Kutta algorithm. As a next step, equations of mass transfer for fuel and radicals are solved with the Crank-Nicholson method of second order approximation in space and time. The initial conditions for the numerical scheme are taken in the form of the travelling wave solution of (7)-(9).

Figures 2 and 3 show the behaviour of the pulsating combustion wave for $\beta=4.08$. This value of $\beta$ is taken slightly above the critical value $\beta \approx 4.0703$ for the Hopf bifurcation. For brevity and clarity, we only show the oscillatory behaviour in terms of the radical concentration (the corresponding profiles for 


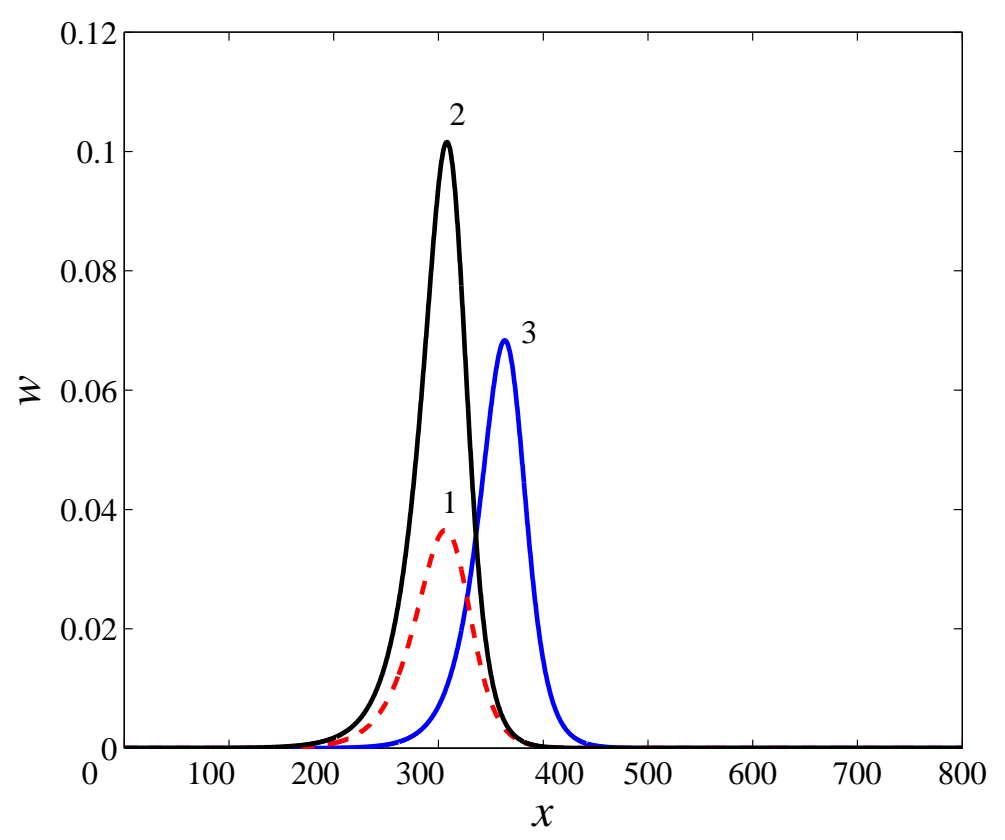

Figure 2: Pulsating combustion wave displaying the profile for the radical concentration $w(x)$ for $\beta=4.08$. Solution profiles are sampled at $t_{1}=0$ (curve 1 ),$t_{2}=8750$ (curve 2) and $t_{3}=17500$ (curve 3 ). 


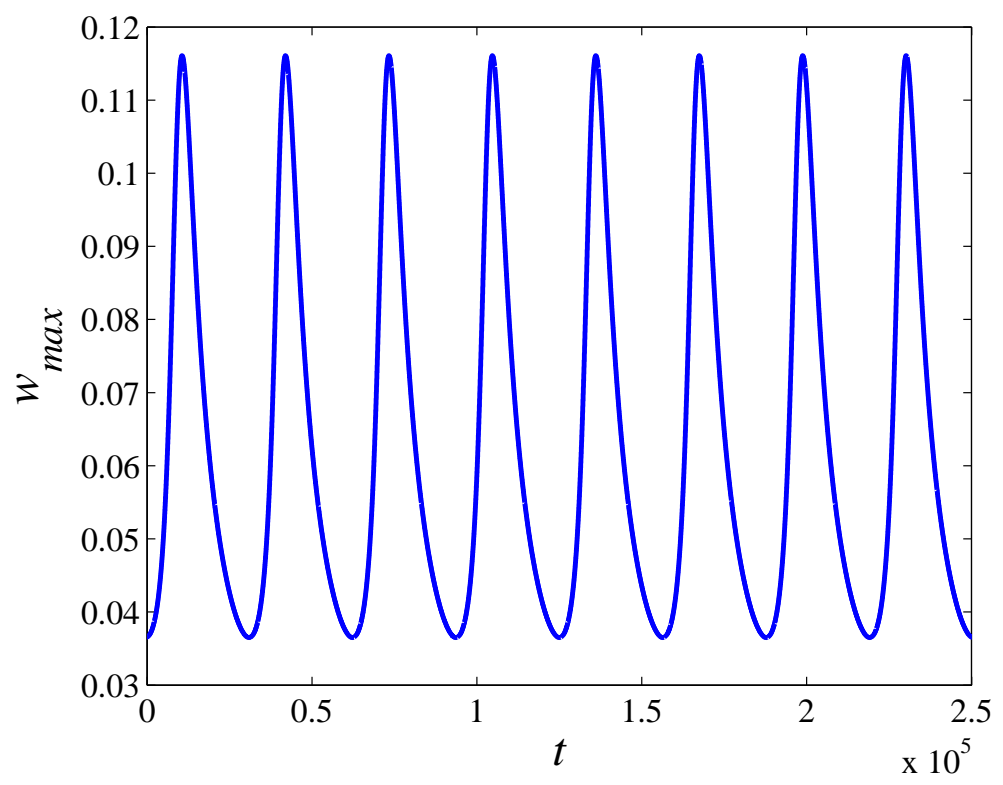

FIGURE 3: The variation of the maximum concentration of the radical $w_{\max }$ with time $\mathrm{t}$ for $\beta=4.08$. 
the fuel concentration and temperature are less distinct in terms of displaying the combustion wave's pulsating behaviour). Figure 2 shows the pulsating wave plotted for three values of time $t_{1}=0, t_{2}=8750$ and $t_{3}=17500$. The period of oscillations was found to be approximately 30492.5. Figure 3 clearly shows the periodic dependence of the maximum value of concentration of the radical with respect to time. In order to clarify the nature of the Hopf bifurcation, the properties of the emerging periodic solution branch were investigated in detail. Parameter $\beta$ is gradually increased beyond the critical value for the Hopf bifurcation $\beta_{h} \approx 4.0703$ to $\beta=4.08$ and the pulsating combustion wave is found by solving the governing partial differential equations (2)-(4) for each value of the parameter $\beta$. The magnitude of oscillations of the maximum value of the radicals concentration, $\Delta w_{\max }=\max \left\{w_{\max }(t)\right\}-\min \left\{w_{\max }(t)\right\}$ over one period $0<\mathrm{t}<\mathrm{T}$, was analysed and the dependence of $\Delta w_{\max }(\beta)$ shows root type behaviour typical for a periodic solution branch emanating from a Hopf bifurcation point. Our analysis shows that the Hopf bifurcation above is of supercritical type.

As $\beta$ increases further a period doubling bifurcation occurs in a manner similar to the one step models considered by Bayliss and Matkowsky [1] and Weber et al. [10]. We found both period two and period four solutions for the current model with chain branching reaction mechanism. These results are illustrated in Figures 4 and 5 respectively for slightly larger values of $\beta$. We conjecture the existence of a sequence of period doubling bifurcations leading to chaotic solutions before extinction. A detailed analysis of this conjecture is currently being investigated. We note that extinction occurred for $\beta \approx 4.11$.

\section{Conclusions}

In this article we undertook a preliminary investigation into the oscillatory behaviour of combustion wave propagation in an adiabatic model with a two step chain branching reaction mechanism. Pulsating solutions with period 


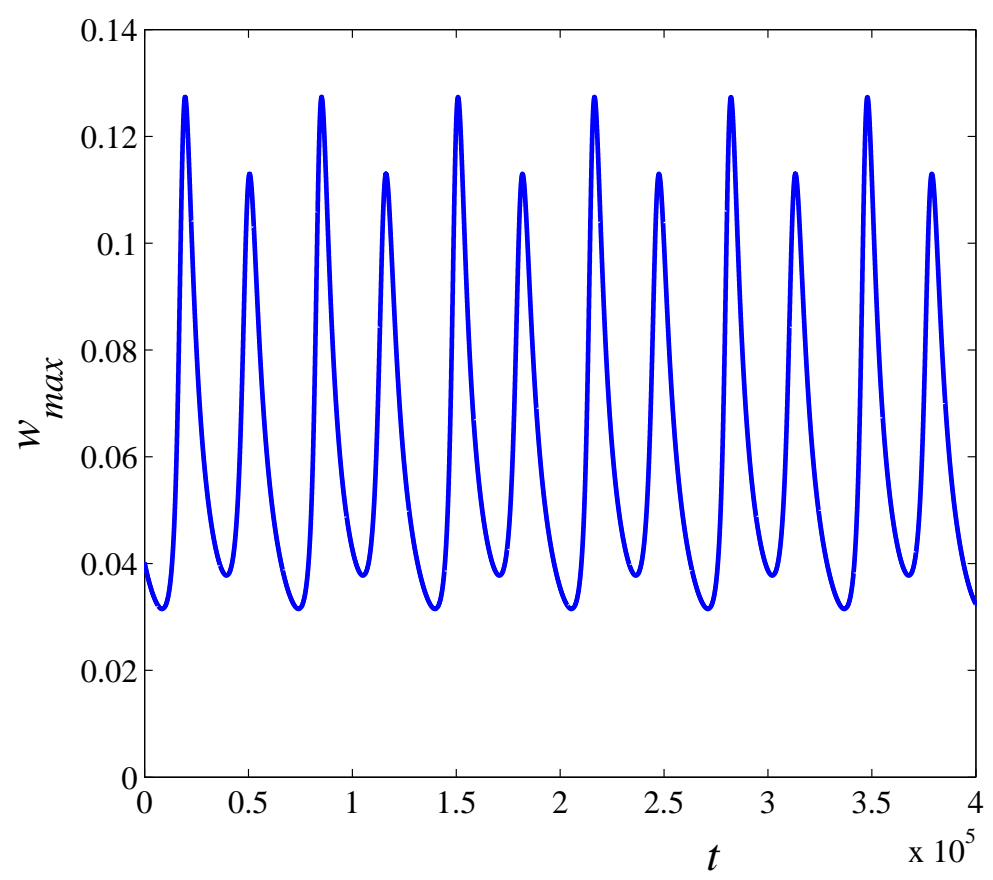

Figure 4: Period-two solution showing the variation of $w_{\max }$ with time $t$ for $\beta=4.0823$. 


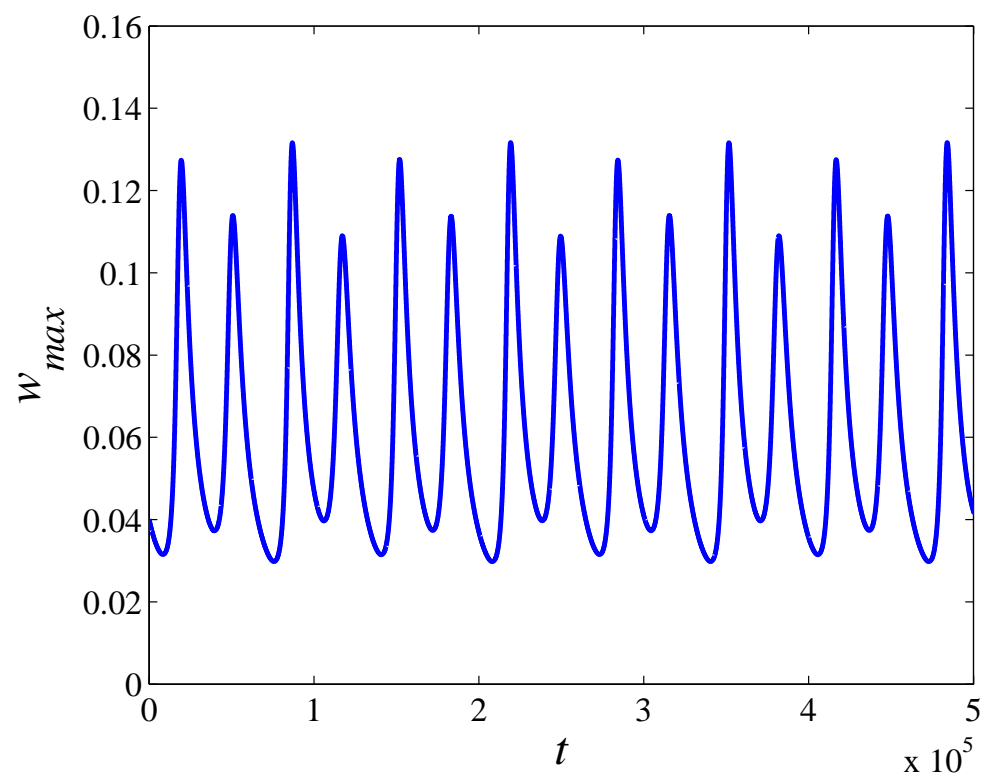

Figure 5: Period-four solution showing the variation of $w_{\max }$ with time $t$ for $\beta=4.0827$. 
two and period four were observed. At this stage it is not clear whether these bifurcations are part of the Feigenbaum period doubling cascade leading to chaos before the extinction of the combustion wave. Although our analysis presented here was for $L_{A}=3$, the above scenario (the existence of a supercritical Hopf bifurcation and the emergence of pulsating solutions from the Hopf bifurcation and the appearance of a period-doubling route to extinction) was the same for all Lewis numbers of the fuel in the range $1<\mathrm{L}_{A}<5$. However, for $L_{A}>5$ we have been unable to detect any oscillatory solutions from the Hopf bifurcation. A possible reason for this is the change in nature of the Hopf bifurcation from supercritical to subcritical through a Bautin bifurcation for $\mathrm{L}_{\mathrm{A}} \approx 5$. This issue needs further clarification and will be investigated in the course of our ongoing work.

\section{References}

[1] A. Bayliss \& B. J. Matkowsky, Two routes to chaos in condensed phase combustion, SIAM J. Appl. Math., 50, 1990, 437-459. http://epubs.siam.org/SIAP/siap_toc.html C1020, C1027

[2] J. W. Dold and R. O. Weber, Reactive-Diffusive stability of planar flames with modified Zeldovich-Liñán kinetics. In: F.J. Higuera, J. Jime'nez and J.M. Vega (Eds), Simplicity, Rigor and Relevance in Fluid Mechanics: A volume in honor of Amable Liñán, CIMNE (Barcelona), 2004. C1018, C1019

[3] C. -G. Feng, Q. -X. Zeng, L. -Q. Wang \& X. Fang, Study of the mechanism of oscillatory solid-phase combustion by a non-linear chemical kinetic model, J. Chem. Soc. Faraday Trans., 1996, 92, 2971-2975. doi:10.1039/FT9969202971 C1020

[4] V. V. Gubernov, G. N. Mercer, H. S. Sidhu and R. O. Weber, Evans function stability of nonadiabatic combustion waves, Proc. $R$. Soc. 
Lond. A, 460, 2004, 2415-2435.doi:10.1098/rspa.2004.1285 C1019, $\mathrm{C} 1021$

[5] V. V. Gubernov, H. S. Sidhu, \& G. N. Mercer, Combustion waves in a model with chain branching reaction and their stability, Combust. Theory Mod., 12, 2008, 407-431. doi:10.1080/13647830701716948 C1019, C1020, C1022

[6] V. V. Gubernov, H. S. Sidhu, \& G. N. Mercer, Combustion waves in a model with chain branching reaction, J. Math. Chem., 39, 2006, 1-14. doi:10.1007/s10910-005-9000-x C1018, C1019

[7] D. W. Mikolaitis, Adiabatic flame speeds and the Zeldovich-Liñán model, Combust. Sci. and Tech., 49, 1986, 277-288.doi:10.1080/00102208608923918 C1018

[8] A. G. Merzhanov and E. N. Rumanov, Physics of reaction waves, Rev. Mod. Phys., 71, 1999, 1173-1211. C1018

[9] H. S. Sidhu, V. V Gubernov and G. N. Mercer, Analysing combustion waves in a model with chain branching, In Geoffry N. Mercer and A. J. Roberts, editors, Proceedings of the 8th Biennial Engineering Mathematics and Applications Conference, EMAC-2007, vol 49 of ANZIAM J., October 2007, pages C100-C115, http://anziamj . austms.org.au/ojs/index.php/ANZIAMJ/article/view/310 C1018, C1019, C1020

[10] R. O. Weber, G. N. Mercer, H. S. Sidhu and B. F. Gray, Combustion waves for gases $(\mathrm{Le}=1)$ and solids $(\mathrm{Le} \rightarrow 1)$, Proc. R. Soc. Lond. A, 453, 1997, 1105-1118. doi:10.1098/rspa.1997.0062 C1020, C1027

\section{Author addresses}

1. H. S. Sidhu, School of Physical, Environmental and Mathematical Sciences, University of New South Wales at the Australian Defence 
Force Academy, Canberra ACT 2600, Australia.

2. V. V. Gubernov, P. N. Lebedev Physical Institute of Russian Academy of Science, Department of Theoretical Physics, Russian Federation.

3. A. V. Kolobov, P. N. Lebedev Physical Institute of Russian Academy of Science, Department of Theoretical Physics, Russian Federation.

4. A. A. Polezhaev, P. N. Lebedev Physical Institute of Russian Academy of Science, Department of Theoretical Physics, Russian Federation.

5. G. N. Mercer, School of Physical, Environmental and Mathematical Sciences, University of New South Wales at the Australian Defence Force Academy, Canberra ACT 2600, Australia.

6. J. J. Sharples, School of Physical, Environmental and Mathematical Sciences, University of New South Wales at the Australian Defence Force Academy, Canberra ACT 2600, Australia. 